\title{
Reactivación de Fallas Geológicas en Respuesta al Cambio de los Esfuerzos en la Corteza: Ejemplos del Sector Oriente de la Sierra de Pachuca
}

\author{
José Cruz Escamilla Casas ${ }^{a^{*}}$, Elvira Meneses Meneses ${ }^{\mathrm{b}}$, Edgar Misael Uribe Alcántara ${ }^{\text {a }}$ \\ a Área Académica de Ciencias de la Tierra y Materiales, Universidad Autónoma del Estado de Hidalgo, Carretera Pachuca-Tulancingo km 4.5, Col. Carboneras, \\ Mineral de la Reforma, Hidalgo, C.P. 42184. \\ ${ }^{b}$ Estudiante de Licenciatura en Ingeniería en Geología Ambiental, Área Académica de Ciencias de la Tierra y Materiales, Universidad Autónoma del Estado de \\ Hidalgo, Carretera Pachuca-Tulancingo km 4.5, Col. Carboneras, Mineral de la Reforma, Hidalgo, C.P. 42184
}

\section{Resumen}

En el presente estudio se muestran los resultados preliminares de los análisis y observaciones realizadas en los planos de falla que afloran en el sector oriental de la Sierra de Pachuca, donde se ubican los cerros La Crucita, La Sotolera y Las Colmenas. En los planos de falla se observaron conjuntos de estrías superpuestos y con diferentes orientaciones. Los conjuntos de estrías se pueden clasificar en dos diferentes orientaciones, las cuales se interpretan como reactivaciones en el movimiento de los bloques de falla en respuesta al cambio de orientaciones de los esfuerzos principales en la corteza. Se hace una comparación entre un sentido de desplazamiento antiguo que define fallas normales y uno más reciente que define fallas de desplazamiento lateral. Los resultados permiten formular la hipótesis de que en la Sierra de Pachuca, se instauró un régimen de trans-tensión muy probablemente de escala regional.

Palabras Clave: Sierra de Pachuca, Reactivación de Fallas, Esfuerzos Principales, Trans-Tensión.

\section{Introducción}

Una de las formas más comunes de deformación en las rocas es la que ocurre cuando un cuerpo rocoso se desliza con respecto a otro a lo largo de zonas o superficies discretas. A estas zonas o superficies discretas de desplazamiento se les denomina fallas geológicas (Lyell, 1871). Price (1966) define a una falla como "un plano o fractura que exhibe evidencia de movimiento diferencial de las masas rocosas a cada lado del plano." Los trabajos mas recientes muestran que las fallas son en realidad zonas, con un volumen que incluye segmentos interactivos y entrelazados, brechas y rocas fracturadas (Childs et al., 2009)

Por otro lado, particularmente para el estudio de cinemática de las fallas, es imprescindible identificar estructuras subordinadas tales como las estrías de falla y otras estructuras de menor orden como son los indicadores cinemáticos (Petit, 1987; Doblas, 1998; Angelier, 1994, Candela et al., 2016). Esto se debe a que las estrías se forman por fricción entre los bloques de roca al momento en que se desplaza uno con respecto del otro.

Desde otro punto de vista, de acuerdo con la hipótesis de Wallace-Bott (Wallace, 1951; Bott, 1959) es ampliamente aceptado que durante el movimiento de los bloques de roca, el vector de desplazamiento es paralelo a la resultante del esfuerzo cortante del mecanismo de la falla. Adicionalmente, el sentido de desplazamiento en una falla puede cambiar debido a las variaciones en el campo de esfuerzos en la corteza superior de la tierra, esto con respecto al tiempo; de ahí que sea posible observar conjuntos de estrías superpuestos en un mismo plano de falla. Consecuentemente, la caracterización de los cambios en los campos de esfuerzos en la corteza implica la identificación de la reactivación de la cinemática de las fallas (Sümer, 2015; Otsubo, 2017).

El objetivo del presente trabajo es la reconstrucción de los campos de paleo esfuerzos en el sector oriental de la Sierra de Pachuca, con base en la colecta sistemática de datos de cinemática en fallas geológicas en los Cerros La Crucita, La Sotolera y Las Colmenas, al oriente del Municipio de Mineral de la Reforma (Figuras 1, 2 y 3).

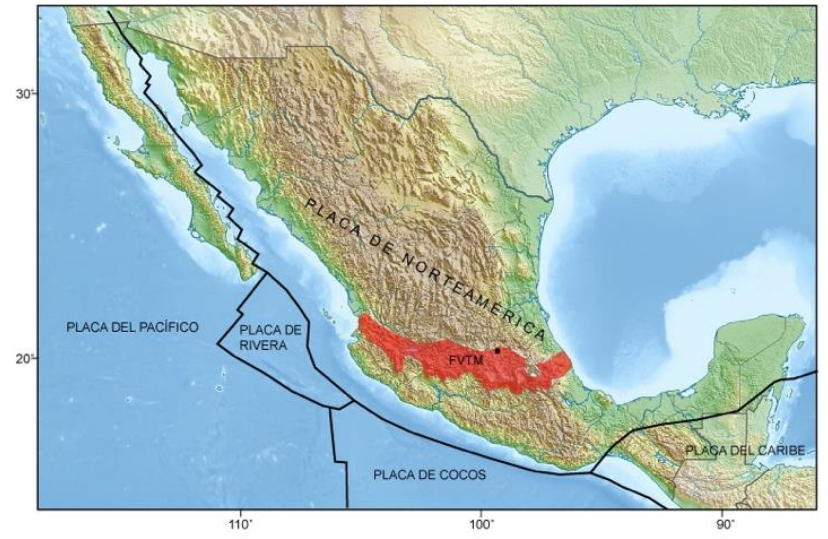

Figura 1. Mapa del territorio mexicano mostrando los principales elementos tectónicos. En rojo se muestra la ubicación de la Faja Volcánica Trans Mexicana. El rectángulo en negro muestra la ubicación del Municipio de Mineral de la Reforma. 
Los datos preliminares del estudio de fallas superpuestas que se presentan y su correspondiente interpretación, permiten formular la hipótesis de que en la zona estudiada hay evidencia de reactivación en las fallas, resultado del cambio sustancial en la orientación de los esfuerzos en la corteza y que, muy probablemente, la zona esté afectada por un régimen de trans-tensión de escala regional.

\section{Geología Local}

La zona de estudio se localiza en la porción norte-oriente del municipio Mineral de la Reforma, Hidalgo. Los positivos topográficos, Cerros la Crucita, La Sotolera y Las Colmenas, tienen una altitud que oscila entre los 2300 y 2480 msnm (Figura 3). El lecho rocoso está compuesto por coladas lávicas y estructuras dómicas de composición dacítica y riolítica, sobreyacidas por coladas piroclásticas en contacto por discordancia angular con intercalaciones de depósitos epiclásticos y horizontes de caliche.

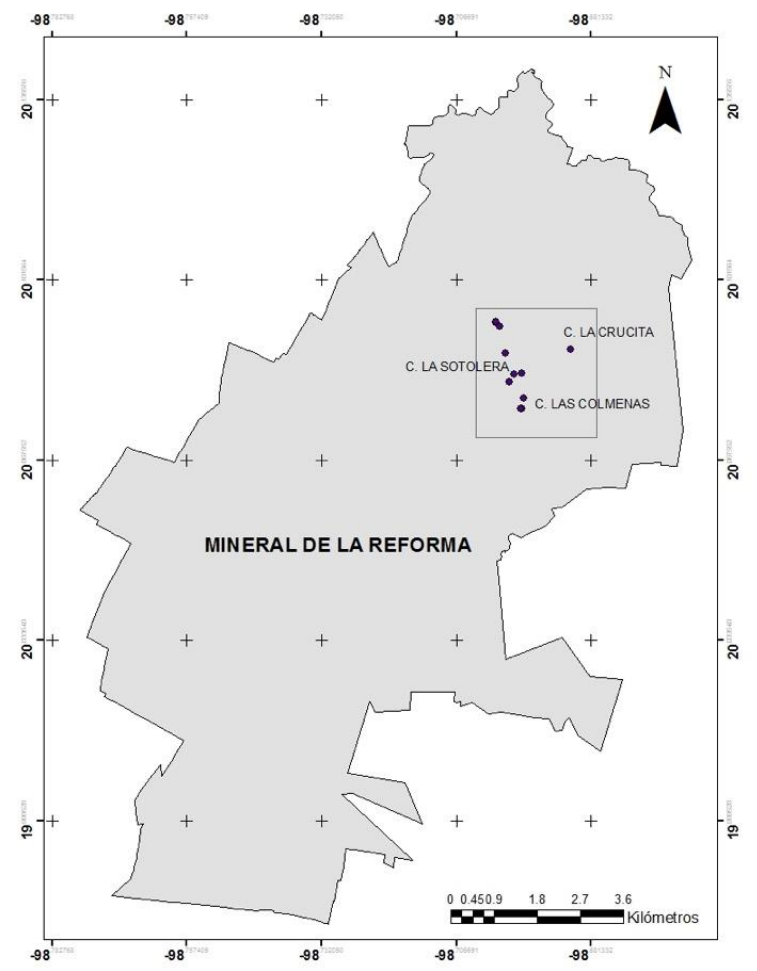

Figura 2: Mapa de localización mostrando la zona de estudio (recuadro). El polígono sombreado corresponde al área del municipio Mineral de la Reforma, Hidalgo. Los puntos negros indican las estaciones de campo.

Hacia la base de los cerros estudiados, aflora un vitrófiro basal color gris pálido que en ocasiones presenta abundante oxidación y silicificación, se caracteriza por la ausencia de fenocristales y su coloración típica. En la parte intermedia de los cerros, predominan riolitas con textura porfirítica, color gris a gris oscuro, con abundantes fenocristales subhedrales de cuarzo y plagioclasa; la roca presenta macro vesículas orientadas paralelamente a los planos de flujo. El color de la roca cambia a gris rojizo por efectos de oxidación. Ocasionalmente se observan fragmentos dispersos de obsidiana negra en la regolita.
Particularmente, el Cerro La Sotolera, aloja un cuerpo intrusivo de pórfido cuarcítico, gris claro y en ocasiones blanco, con una orientación preferencial E-W. El pórfido cuarcítico presenta abundantes cristales de cuarzo subhedrales a anhedrales, de color gris claro a gris, embebidos en una matriz de cuarzo y feldespato hipohialina a hipocristalina. Ocasionalmente, el cuerpo intrusivo presenta oxidación en los planos de fractura y hacia los bordes con la roca encajonante. En las partes topográficamente altas, en la porción norte del Cerro La Sotolera y la cima del Cerro la Crucita, aflora una brecha volcánica constituida por fragmentos del pórfido cuarcítico, embebidos en una matriz predominantemente riolítica y con abundante oxidación. En las faldas de los cerros y en las depresiones locales afloran las coladas piroclásticas y depósitos epiclásticos. Por su posición estratigráfica, se asume que los depósitos piroclásticos y epiclásticos corresponden a las unidades volcánicas más jóvenes.

A pesar de que actualmente las edades de las rocas estudiadas no han sido determinadas, McKee et al., 1992 reportaron edades de K-Ar $20.3 \pm 0.5 \mathrm{Ma}$ para la mineralización hidrotermal en el Distrito Minero PachucaReal del Monte, alojado en la Sierra de Pachuca. Así mismo, estos autores establecieron que en la Sierra de Pachuca, las rocas volcánicas, diques, y otras rocas intrusivas, la alteración hidrotermal, mineralización y posiblemente ciertas fallas parecen estar íntimamente asociadas en tiempo y espacio y que, a su vez, son parte del sistema volcánico activo del Mioceno temprano.

\section{Materiales y Métodos}

Se utilizó una brújula tipo Brunton para colectar datos estructurales en planos y estrías de falla en 11 estaciones de campo (Figura 3). La localización de las estaciones de campo y la referencia geográfica correspondiente se obtuvieron mediante una unidad portátil GPS Spectra - Global Mapper 20. Los datos estructurales que se colectaron en cada estación son: para cada plano de la falla, el rumbo y "echado"; y para las estrías, los valores de dirección e inclinación. La resolución de la cinemática entre los bloques se basó en la identificación de las estructuras menores y criterios descritos por Petit, (1987) y Doblas, (1998); esto para determinar la cinemática diferente en cada uno de los episodios de fracturamiento observados.

El programa computacional ArcMap se empleó para la manipulación de los datos geográficos de ubicación de las estaciones de campo y para la proyección de la base topográfica del área, para generar el modelo de elevación digital. Los datos estructurales se analizaron utilizando el programa computacional FaultKin y se proyectaron en el hemisferio sur de la red estereográfica equiareal.

En este estudio, se aplicó el método de los ejes $\mathrm{P}$ y T ( $\mathrm{P}=$ acortamiento y $\mathrm{T}=$ extensión) para determinar la orientación de los esfuerzos principales en la corteza, descrito por Marrett y Allmendinger (1990). En los gráficos se proyectan en la red estereográfica equiareal los planos de falla, las estrías de falla, los sentidos de movimiento de los bloques, los esfuerzos principales $\left(\sigma_{1}, \sigma_{2}\right.$, y $\left.\sigma_{3}\right)$ y los sectores correspondientes a los dominios de $\mathrm{P}$ y $\mathrm{T}$ (segmentos claros y sombreados, respectivamente). Los sectores $\mathrm{P}$ y $\mathrm{T}$ están delimitados por las 
dos fallas que son las posibles soluciones al sistema de esfuerzos. Así mismo, los ejes $\mathrm{P}$ y $\mathrm{T}$ tienen orientación equivalente a la de los esfuerzos $\sigma_{1}$ y $\sigma_{3}$, respectivamente.

La aplicación del método de los ejes $\mathrm{P}$ y $\mathrm{T}$ se justifica porque facilita la determinación de la orientación de los esfuerzos y facilita la interpretación de datos de estructuras superpuestas.

\section{Resultados y Discusión}

En este trabajo, se identificó la superposición de dos conjuntos de estrías de falla con diferentes orientaciones y contenidas en el mismo plano. En general, los planos de falla con estrías superpuestas se consideran como el resultado de diferentes etapas de deformación debido a la variación de las condiciones de los esfuerzos tectónicos (e.g. Kaymakci et al., 2000; Otsubo et al., 2009). Por otro lado, las rocas estudiadas presentan un estado de preservación óptimo de las estructuras, mismas que permiten la identificación de las estructuras menores asociadas con la cinemática de las fallas.

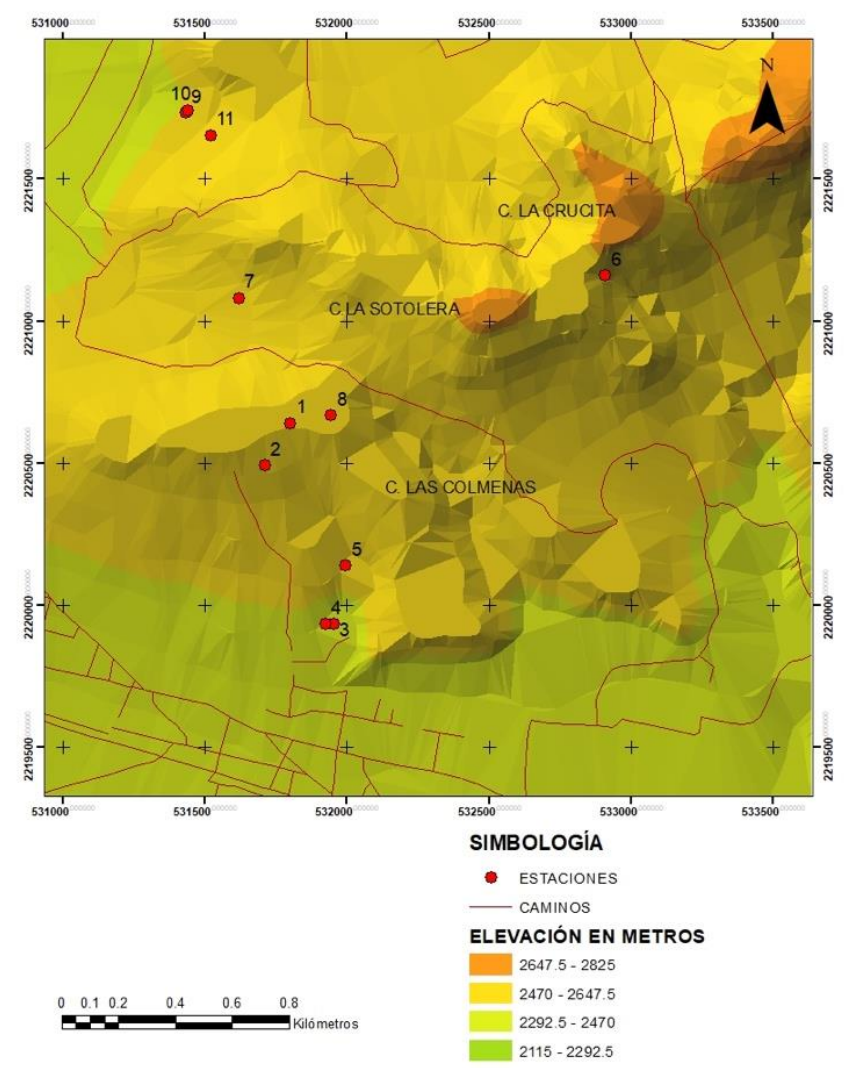

Figura 3: Modelo digital de elevación mostrando la ubicación de las estaciones de campo y de los cerros La Sotolera, Crucita y Las Colmenas.

En la Tabla 1, se muestran los datos de campo correspondientes a la actitud geológica (registro de rumbo y echado) para cada una de los planos de falla considerados en este análisis. En las estaciones en donde se observan planos de falla con conjuntos de estrías diferentes se les ha etiquetado como desplazamiento superpuesto.

La observación en campo permite identificar la reactivación de fallas, tal y como se acusa por la presencia de dos conjuntos de estrías superpuestos en el mismo plano de falla (Figura 4).
Con la finalidad de caracterizar la reactivación de las fallas, los datos estructurales se separaron en dos grupos: uno corresponde al episodio más antiguo en donde las fallas normales son predominantes (Figura 5 y Tabla 2), y el más reciente, que generó fallas de desplazamiento lateral (Figura 6 y Tabla 3)

Tabla 1: Datos estructurales de los planos y tipos de falla. Referirse a la Figura 3 para la localización de las estaciones.

\begin{tabular}{cccc}
\hline Estación & Rumbo & Echado & Desplazamiento \\
\hline 1 & 324 & 36 & normal \\
2 & 331 & 36 & oblicuo \\
3 & 276 & 84 & superpuesto \\
3 & 246 & 80 & lateral \\
4 & 87 & 38 & normal \\
5 & 144 & 43 & oblicuo \\
6 & 319 & 73 & normal \\
6 & 337 & 86 & oblicuo \\
6 & 334 & 78 & oblicuo \\
7 & 137 & 68 & oblicuo \\
8 & 103 & 82 & oblicuo \\
9 & 292 & 42 & superpuesto \\
10 & 187 & 37 & superpuesto \\
11 & 330 & 29 & superpuesto \\
\hline
\end{tabular}

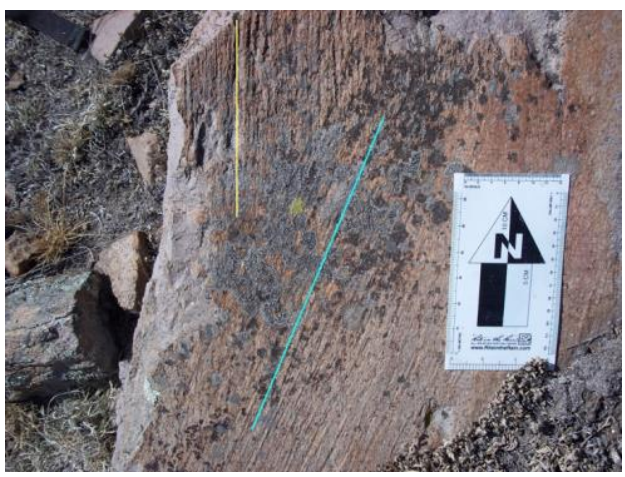

Figura 4: Fotografía apuntando al norte de un plano de falla de la Estación 3. En la estructura concurren dos conjuntos de estrías: el más antiguo define líneas verticales (parcialmente obliteradas), mientras que el más reciente define líneas oblicuas. 


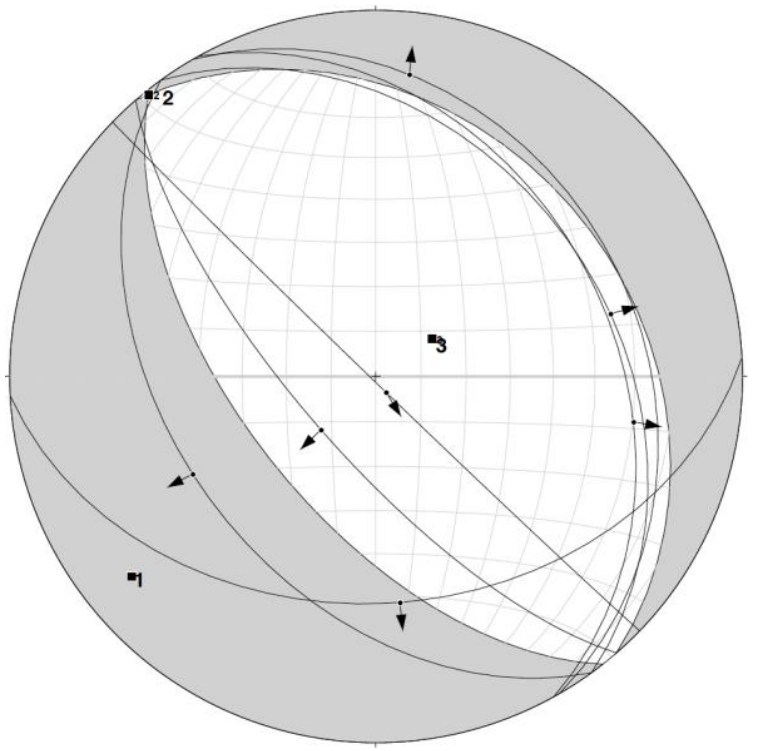

Figura 5: Proyección estereográfica en la red equiareal, hemisferio inferior, de las 7 fallas normales del episodio más antiguo. Los números 1 , 2 y 3 corresponden a la orientación de los ejes principales $(\sigma 1, \sigma 2$, y $\sigma 3)$, respectivamente. Los segmentos sombreados corresponden al cuadrante $\mathrm{T}$, los claros al P.

Tabla 2: Dirección e inclinación, en grados, de los esfuerzos principales y de los ejes $\mathrm{P}$ y $\mathrm{T}$ del episodio más antiguo.

\begin{tabular}{ccc}
\hline Eje & Dirección & Inclinación \\
\hline 1 & 230.8 & 14.9 \\
2 & 321.1 & 1.2 \\
3 & 55.6 & 75.1 \\
$\mathrm{P}$ & 55.6 & 75.1 \\
$\mathrm{~T}$ & 230.8 & 14.9 \\
\hline
\end{tabular}

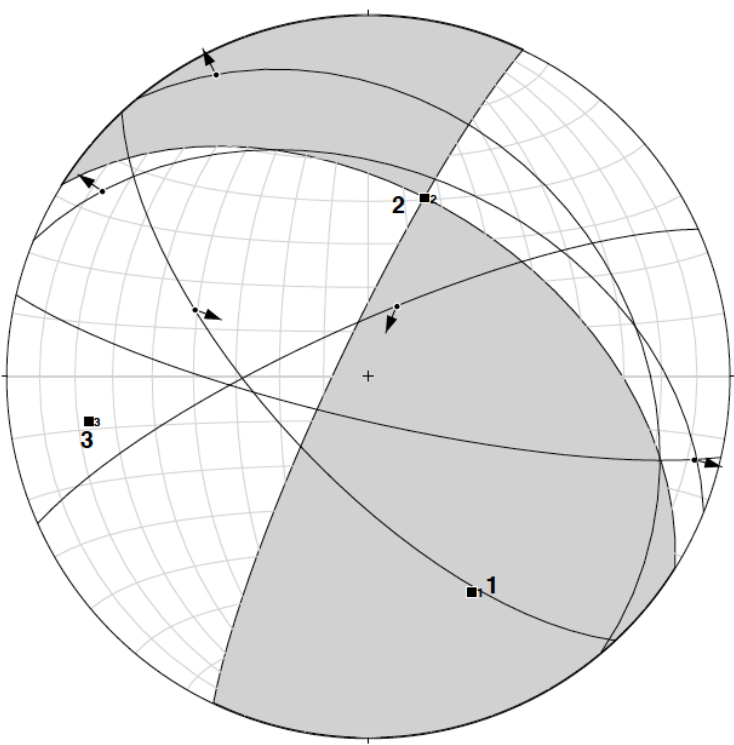

Figura 6: Proyección estereográfica en la red equiareal, hemisferio inferior, de las 5 fallas laterales del episodio más reciente. Los números 1, 2 y 3 corresponden a la orientación de los ejes principales $(\sigma 1, \sigma 2$, y $\sigma 3)$, respectivamente. Los segmentos sombreados corresponden al dominio $\mathrm{T}$, los claros al P.
Tabla 3: Dirección e inclinación, en grados, de los esfuerzos principales y de los ejes $\mathrm{P}$ y $\mathrm{T}$ del episodio más reciente.

\begin{tabular}{ccc}
\hline Eje & Dirección & Inclinación \\
\hline 1 & 154.5 & 34.3 \\
2 & 17.4 & 47 \\
3 & 261 & 22.6 \\
$\mathrm{P}$ & 261 & 22.6 \\
$\mathrm{~T}$ & 154.5 & 34.3 \\
\hline
\end{tabular}

Los resultados obtenidos del episodio más antiguo se muestran en la Tabla 2 y en la Figura 5. A pesar de que las fallas consideradas en este grupo son normales, algunas tienen componente lateral. Consecuentemente, la resolución del sistema comprende dos posibles soluciones: una, con una componente de falla normal en combinación con un desplazamiento lateral izquierdo y dos, componente de falla inversa con desplazamiento lateral derecho, Figura 5.

Las orientaciones de los esfuerzos para el episodio más reciente se muestran en la Tabla 3 y en la Figura 6. En este episodio predominan las fallas de desplazamiento lateral. La resolución del sistema comprende dos posibles soluciones: a) componente de falla inversa combinado con un desplazamiento lateral derecho y b) un componente de falla normal combinado con un desplazamiento lateral izquierdo.

$\mathrm{Si}$ se considera que ambos episodios corresponden a un continuo pulsante en la deformación de la corteza, en el primer episodio predomina la extensión y en el más reciente el desplazamiento oblicuo (combinación de lateral con normal). Consecuentemente, en conjunto ambos episodios constituyen un sistema de trans-tensión.

\section{Conclusión}

El estudio de la cinemática en fallas en la zona de estudio, ha permitido caracterizar con más detalles la deformación frágil de la Sierra de Pachuca. En este estudio, se presenta la evidencia de la reactivación de fallas, en el sector oriental de la Sierra, mediante datos obtenidos en campo que permiten sugerir la presencia de dos episodios de deformación (Tabla 2 y 3). Adicionalmente, considerando que la deformación frágil de las rocas estudiadas ocurrió en algún tiempo cercano a los $20 \mathrm{Ma}$, nuestros resultados muestran un cambio en la dirección de movimiento de los bloques de falla ocurridos durante un intervalo relativamente corto. Finalmente, la interpretación de los resultados obtenidos, permite formular la hipótesis de que en la Sierra de Pachuca se instauró un régimen de trans-tensión que pudiera tener dimensiones de escala regional. Sin embargo, es primordial expandir el estudio a otros sectores de la Sierra para poder validar la hipótesis propuesta.

\section{English Summary}

Reactivation of Geologic Faults in Response to Changes in Crustal Stress: Examples From Eastern Sierra de Pachuca

\section{Abstract}


In this work, we present preliminary results from observations and structural analyses made on outcropping fault planes in the eastern sector of the Sierra de Pachuca, where La Crucita, La Sotolera, and Colmenas hills, are located. On the studied fault planes, several sets of superposed slickenlines with different orientation were observed. The orientations of the slickenlines can be grouped into two different sets, here interpreted as reactivation of faults in response to changes in the orientation of crustal stress. We compared the older dipslip normal faulting with the younger oblique-slip faulting. Our results support the working hypothesis that the Sierra de Pachuca was, very likely, affected by a regional transtensional regime.

\section{Keywords:}

Sierra de Pachuca, Reactivated Faults, Principal Stresses, Trans-Tension

\section{Agradecimientos}

Este trabajo ha sido realizado durante el desarrollo de los proyectos de investigación: "Caracterización en un Espacio Bidimensional de Fallas y Fracturas en la Sierra de Pachuca" y del proyecto "Desarrollo de una metodología para la Creación de mapas de riesgo estatales por procesos de Remoción de Masa con base en Sistemas de Información Geográfica". Los autores agradecen a dos revisores anónimos, cuyos comentarios y sugerencias ayudaron a enriquecer $\mathrm{y}$ mejorar el presente trabajo.

\section{Referencias}

Angelier. J., 1994. Fault slip Analysis and palaeostress reconstruction. En: Continental Deformation (ed. P. L. Hancock): 53-100. Pergamon Press, University of Bristol, London.

Bott, M.H.P., 1959. The mechanics of oblique slip faulting. Geology Magazine, 96, 109-117.

Doblas, M., 1998. Slickenside kinematic indicators. Tectonophysics, 295, 187-197.

Candela, T., Brodsky, E.E., 2016. The minimum scale of grooving on faults. Geology 44, 8, 603-606.

Childs, C., Manzocchi, T., Walsh, J. J., Bonson, C. G., Nicol, A., Schöpfer, M. P., 2009. A geometric model of a fault zone and fault thickness variations. Journal of Structural Geology 31, 117-127.

Kaymacki, N., White, S. H., van Dijk, P. M., 2000. Paleostress inversion in a multiphase deformed area: kinematic and structural evolution of the Cankiri Basin (central Turkey), Part I. En: Bozkurt, E., Winchester, J. A., Piper, J. A. D., (Editores), Tectonics and Magmatism in Turkey and the Surrounding Area. 445-473. Geological Society of London Special Publication No. 173.

McKee, E. H., Dreier, J. E., Noble, D. C., 1992, Early Miocene Hydrothermal Activity at Pachuca-Real del Monte, Mexico: An Example of Space-Time Association of Volcanism and epithermal Ag$\mathrm{Au}$ Vein Mineralization. Economic Geology, 87, 1635-1637.

Petit, J.P., 1987. Criteria for the sense of movement on fault surfaces in brittle rocks. Journal of Structural Geology 9, 56, 597-608.

Price, N.J., 1966. Fault and Joint Development in Brittle and Semi-Brittle Rock. Pergamon, Oxford

Lyell, C., 1871. The Student's elements of Geology: New York, Harper \& Brothers Publishers, $640 \mathrm{p}$

Marrett, R.A., Allmendinger, R.W., 1990. Kinematic Analysis of fault-slip data, Journal of Structural Geology 12, 973-986.

Otsubo, M., Shigematsu, N.,Kitagawa, Y., Koizumi, N., 2009. Stress history in the forearc region of the Nankai trough subduction zone: paleostress analysis based on faults in core samples from the Kumano Ichiura and Kihoku Miyama sites, Kii Peninsula, SW Japan. Journal of the Geological Society of Japan. 115, 457-469.

Otsubo, M., 2017. Reactivation of map-scale faults in response to changes in crustal stress: Examples from Boso Peninsula, Japan. Quaternary International 450, 117-124.

Sümer, Ö, 2015. Evidence for the reactivation of a pre-existing zone of weakness and its contributions to the evolution of the Küçük Menders Graben: a study of the Ephesus fault, western Anatolia, Turkey. Geodinamica Acta 27, 2-3, 129-152.

Wallace, R. E., 1951. Geometry of shearing stress and relationship to faulting. Journal of Geology 59, 111-130. 